\title{
EMISSION OF FERMIONS IN LITTLE STRING THEORY
}

\author{
OSCAR LORENTE-EsPÍN \\ Departament de Física i Enginyeria Nuclear, Universitat Politècnica de Catalunya, \\ Comte Urgell, 187, E-08036 Barcelona, Spain.
}

\begin{abstract}
It is well known that little string theory (LST) black holes radiate a purely thermal spectrum of scalar particles. This theory lives in a Hagedorn phase with a fixed Hagedorn temperature that does not depend on its mass. Therefore the theory keeps a thermal profile even taking into account self-gravitating effects and the backreaction of the metric. This has implications concerning the information loss paradox; one would not be able to recover any information from the LST black hole since the emission of scalar particles is totally uncorrelated. Several studies of the emission spectrum in LST concern scalar fields; it is our aim in this work to extend the study to the emission of fermions in order to verify that the most relevant conclusion for the scalar field remains valid for the fermion fields. Thus, we have calculated the emission probability, the flux and also the greybody factor corresponding to a fermion field in LST background.
\end{abstract}

KEYWORDS: Black Holes, Hawking radiation, Fermions.

E-mail: oscar.lorente-espin@upc.edu 


\section{Contents}

1 Introduction $\quad 1$

2 Description of the model 2

3 Tunneling of fermions in LST 3

3.1 Emission probability ......................... 4

3.2 Fermion modes and backscattering spectrum . . . . . . . . . . . 7

\begin{tabular}{|l|l|} 
& 13 \\
\hline
\end{tabular}

\section{INTRODUCTION}

In [1] Hawking demonstrated that black holes radiate a purely thermal radiation at a definite temperature. This fact drove us to the so-called information loss paradox, which states the violation of quantum unitary evolution. A lot of work has been done in order to solve this paradox, e.g. great success has been achieved in string theory framework using holography principle techniques [2, 3, 4, 5].

On the other hand, using semiclassical techniques, some authors in [6, 7, 8, 9, 10] have showed how deviations of the black hole radiance from purely a thermal spectrum enable us to recover the information lost. In the work of Hawking it was not considered the self-gravitation of the emitted particles neither the backreaction of the background metric. Nevertheless, the tunneling picture proposed in [6] takes into account the self-gravitation of the radiation; hence imposing energy conservation the metric backreacts when the black hole emits and the event horizon shrinks. The tunneling mechanism matches the heuristic picture more considered more closely, namely the creation of a particle-antiparticle pair just outside the horizon or inside of the black hole. From a pair created outside the horizon one member of the pair, e.g. the antiparticle, can tunnels through the horizon falling down the hole whereas the particle escapes out. Concretely this flux is detected as Hawking radiation by an asymptotic observer. In the same way, if the pair is created inside the horizon, the particle can tunnel through the horizon escaping to the asymptotic, whereas the antiparticle falls down to the black hole. Anyway, the flux energy of the incoming antiparticles is negative while the outgoing flux of particles is positive. Therefore, taking into account the energy conservation, the total ADM mass is conserved whereas the black hole mass decreases by the amount $M \rightarrow M-\omega$, where $\omega$ is the energy of the emitted particle. As the event horizon 
radius is proportional to the mass, the black hole will be shrunk. It is noteworthy to say that in this tunneling mechanism is the emitted particle which creates the potential barrier [8].

In [11, 12] we studied the Hawking radiation for NS5 and little string theory (LST) black holes using different semiclassical methods, i.e. the tunneling and the complex path method. We verified that the NS5 model showed a nonthermal profile whereas LST showed a thermal behavior. This last conclusion matches the Hagedorn properties of LST, namely the temperature of LST corresponds to the Hagedorn temperature. However, we only studied the emission of a scalar field. It would be interesting to extend the study of the Hawking emission by LST to fermion fields and verify if the LST spectrum changes or remains purely thermal.

The paper is organized as follows. In Sec. II, we briefly summarize some properties and thermodynamics of LST. In Sec. III, we solve the Dirac equation for a fermion field in LST background. Next, we study the emission probability of fermions. Also, we calculate the spectrum and the radiation flux corresponding to a massless fermion field, and we analyze the implications of considering the energy conservation and the backreaction of the metric when the black hole emits. Finally, in Sec. IV, we discuss the most relevant results.

\section{DesCRIPTION OF THE MODEL}

LST 1 is a nongravitational six dimensional and nonlocal field theory [13, 14, 15, 19, 20], believed to be dual to a string theory background, defined as the decoupled theory on a stack of N NS5-branes. In the limit of a vanishing asymptotic value for the string coupling $g_{s} \rightarrow 0$, keeping the string length $l_{s}$ fixed while the energy above extremality is fixed, i.e. $\frac{E}{m_{s}}=$ fixed, the processes in which the modes that live on the branes are emitted into the bulk as closed strings are suppressed. The theory becomes free in the bulk, but strongly interacting on the brane. In this limit, the theory reduces to the little string theory or more precisely to $(2,0)$ LST for type IIA NS5-branes and to $(1,1)$ LST for type IIB NS5-branes [18].

The throat geometry corresponding to N coincident nonextremal NS5-branes in the string frame [21] is

$$
d s^{2}=-f(r) d t^{2}+\frac{A(r)}{f(r)} d r^{2}+A(r) r^{2} d \Omega_{3}^{2}+\sum_{j=1}^{5} d x_{j}^{2},
$$

\footnotetext{
${ }^{1}$ For good reviews on LST and NS5 we address the readers to [13, 14, 15, 16, 17, 18.
} 
where $d x_{j}^{2}$ corresponds to flat spatial directions along the 5 -branes, $d \Omega_{3}^{2}$ corresponds to 3 sphere of the transverse geometry and the dilaton field is defined as

$$
e^{2 \Phi}=g_{s}^{2} A(r)
$$

The metric functions are

$$
f(r)=1-\frac{r_{0}^{2}}{r^{2}} \quad, \quad A(r)=\chi+\frac{N}{m_{s}^{2} r^{2}},
$$

where $r_{0}$ is the location of the event horizon. We define the parameter $\chi$ which takes the values 1 for NS5 model and 0 for LST; these are only the values for which exists a supergravity solution. In addition to the previous fields, there is a $N S-N S H_{(3)}$ form along the $S^{3}, H_{(3)}=2 N \epsilon_{3}$. According to the holographic principle, the high spectrum of this dual string theory should be approximated by a certain black hole in the background (2.1). The geometry transverse to the 5-branes is a long tube which opens up into the asymptotic flat space with the horizon at the other end. In the limit $r \rightarrow r_{0}$ appears the semi-infinite throat parametrized by (t,r) coordinates. In this region the dilaton grows linearly pointing out that gravity becomes strongly coupled far down the throat. The string propagation in

this geometry should correspond to an exact conformal field theory [22]. The boundary of the near horizon geometry is $R^{5,1} \times R \times S^{3}$.

\section{TunNeling OF FERMiOns In LST}

In this section we will study the tunneling of fermions through the event horizon of the LST background (2.1). We write the covariant Dirac equation in a general background [23] for a spinor field $\Psi$,

$$
\left(\gamma^{a} e_{a}^{\mu}\left(\partial_{\mu}+\Gamma_{\mu}\right)+m\right) \Psi=0
$$

where $m$ is the bare mass of the particle; $e_{a}^{\mu}$ are the vielbein defined by the relation $g_{\mu \nu}=$ $\eta_{a b} e_{\mu}^{a} e_{\nu}^{b}$ with $\eta_{a b}=\operatorname{diag}(-1,1,1,1, \ldots)$; and the latin indexes run for local inertial flat coordinates $(0,1,2, \ldots)$, whereas the greek indexes run for general coordinates $(t, r, \theta, \ldots)$. In LST, the vielbein take the form

$$
e_{\mu}^{a}=\operatorname{diag}\left(\sqrt{f(r)}, \sqrt{\frac{A(r)}{f(r)}}, r \sqrt{A(r)}, r \sqrt{A(r)} \sin \theta, r \sqrt{A(r)} \sin \theta \sin \varphi, 1, \ldots, 1\right) .
$$

The spin connection is defined as

$$
\Gamma_{\mu}=\frac{1}{8}\left[\gamma^{c}, \gamma^{b}\right] e_{c}^{\nu} \nabla_{\mu} e_{b \nu}
$$


where $\nabla_{\mu} e_{b \nu}=\partial_{\mu} e_{b \nu}-\Gamma_{\mu \nu}^{\lambda} e_{b \lambda}$ is the covariant derivative of $e_{b \nu}$ and $\left[\gamma^{c}, \gamma^{b}\right]$ the commutator of the gamma matrices. We choose for the gamma matrices [24],

$$
\gamma^{0}=\left(\begin{array}{cc}
-i & 0 \\
0 & i
\end{array}\right) \quad, \quad \gamma^{k}=\left(\begin{array}{cc}
0 & -i \sigma^{k} \\
i \sigma^{k} & 0
\end{array}\right) \quad, \quad k=1,2,3
$$

where the matrices $\sigma^{i}$ are the Pauli matrices,

$$
\sigma^{1}=\left(\begin{array}{ll}
0 & 1 \\
1 & 0
\end{array}\right) \quad, \quad \sigma^{2}=\left(\begin{array}{cc}
0 & -i \\
i & 0
\end{array}\right) \quad, \quad \sigma^{3}=\left(\begin{array}{cc}
1 & 0 \\
0 & -1
\end{array}\right)
$$

The gamma matrices satisfies the Clifford algebra,

$$
\begin{aligned}
& {\left[\gamma^{a}, \gamma^{b}\right]=-\left[\gamma^{b}, \gamma^{a}\right] \quad \text { if } \mathrm{a} \neq \mathrm{b},} \\
& {\left[\gamma^{a}, \gamma^{b}\right]=0 \quad \text { if } \mathrm{a}=\mathrm{b},} \\
& \left\{\gamma^{\mu}, \gamma^{\nu}\right\}=2 g^{\mu \nu} \\
& \left\{\gamma^{a}, \gamma^{b}\right\}=2 \eta^{a b}
\end{aligned}
$$

Thus, taking into account all the aforesaid definitions and properties, we write the full tendimensional Dirac equation for the spinor field in the LST background,

$$
\begin{aligned}
& \left(\gamma^{0} \frac{1}{\sqrt{f(r)}} \partial_{t}+\gamma^{1}\left(\frac{3 A(r)^{\prime} \sqrt{f(r)}}{4 A(r)^{\frac{3}{2}}}+\frac{6 \sqrt{f(r)}}{4 r \sqrt{A(r)}}+\frac{f(r)^{\prime}}{4 \sqrt{A(r) f(r)}}+\sqrt{\frac{f(r)}{A(r)}} \partial_{r}\right)+\right. \\
& +\gamma^{2} \frac{1}{r \sqrt{A(r)}}\left(\cot \theta+\partial_{\theta}\right)+\gamma^{3} \frac{1}{r \sqrt{A(r)}} \sin \theta \partial_{\varphi}+\gamma^{4} \frac{1}{r \sqrt{A(r)}} \sin \theta \sin \varphi \partial_{\psi}+ \\
& \left.+\sum_{j=5}^{9} \gamma^{j} \partial_{x_{j}}+m\right) \Psi=0,
\end{aligned}
$$

where prime denotes derivative with respect to the $r$ coordinate.

\subsection{EMISSION PROBABILITY}

Henceforth, in order to study the probability emission of fermions, we will be interested in the $r-t$ sector of the LST metric, 2

$$
d s_{e f f}^{2}=-f(r) d t^{2}+\frac{A(r)}{f(r)} d r^{2} .
$$

\footnotetext{
${ }^{2}$ In [12], the reduction from the ten-dimensional to the two-dimensional metric of LST is shown.
} 
Working with this effective two-dimensional metric, the Dirac equation corresponding to a spinor field is simplified to

$$
\left(\gamma^{0} \frac{1}{\sqrt{f(r)}} \partial_{t}+\gamma^{1}\left(\frac{f(r)^{\prime}}{4 \sqrt{A(r) f(r)}}+\sqrt{\frac{f(r)}{A(r)}} \partial_{r}\right)+m\right) \Psi=0 .
$$

Taking into account the appropriate choice of the gamma matrices (3.4), we use for the spin-up and spin-down Dirac fields, respectively, the following Wentzel-Kramers-Brillouin (WKB) ansatz [25]:

$$
\begin{aligned}
\Psi_{\uparrow} & =\left(\begin{array}{c}
\mathcal{A}(t, r) \xi_{\uparrow} \\
0 \\
0 \\
\mathcal{D}(t, r) \xi_{\uparrow}
\end{array}\right) \exp \left[\frac{i}{\hbar} S_{\uparrow}(t, r)\right], \\
\Psi_{\downarrow} & =\left(\begin{array}{c}
0 \\
\mathcal{B}(t, r) \xi_{\downarrow} \\
\mathcal{C}(t, r) \xi_{\downarrow} \\
0
\end{array}\right) \exp \left[\frac{i}{\hbar} S_{\downarrow}(t, r)\right],
\end{aligned}
$$

where $S$ is the classical action, whereas $\mathcal{A}, \mathcal{B}, \mathcal{C}$ and $\mathcal{D}$ are arbitrary functions of the coordinates. Measuring the spin in the $z$ direction, the eigenvector of $\sigma^{3}$ for the spin-up and spin-down fields, respectively, are $\xi_{\uparrow}=\left(\begin{array}{l}1 \\ 0\end{array}\right)$ and $\xi_{\downarrow}=\left(\begin{array}{l}0 \\ 1\end{array}\right)$. We will only solve the spin-up case and the spin-down case is solved analogously. Thus, we substitute the spinorup field (3.10) and the gamma matrices $\gamma^{0}$ and $\gamma^{1}$ into the Dirac equation (3.9). Next, we apply the WKB approximation neglecting the $\hbar$ dependent terms, and after some algebra we eventually obtain the following set of equations for the spin-up case:

$$
\begin{gathered}
\left(-\frac{1}{\sqrt{f(r)}} \partial_{t} S_{\uparrow}(t, r)+m\right) \mathcal{A}(t, r)-\sqrt{\frac{f(r)}{A(r)}} \partial_{r} S_{\uparrow}(t, r) \mathcal{D}(t, r)=0, \\
\sqrt{\frac{f(r)}{A(r)}} \partial_{r} S_{\uparrow}(t, r) \mathcal{A}(t, r)+\left(\frac{1}{\sqrt{f(r)}} \partial_{t} S_{\uparrow}(t, r)+m\right) \mathcal{D}(t, r)=0 .
\end{gathered}
$$

For the spin-down case, we would obtain

$$
\begin{gathered}
\left(-\frac{1}{\sqrt{f(r)}} \partial_{t} S_{\downarrow}(t, r)+m\right) \mathcal{B}(t, r)-\sqrt{\frac{f(r)}{A(r)}} \partial_{r} S_{\downarrow}(t, r) \mathcal{C}(t, r)=0, \\
\sqrt{\frac{f(r)}{A(r)}} \partial_{r} S_{\downarrow}(t, r) \mathcal{B}(t, r)+\left(\frac{1}{\sqrt{f(r)}} \partial_{t} S_{\downarrow}(t, r)+m\right) \mathcal{C}(t, r)=0 .
\end{gathered}
$$


In order to obtain nonvanishing values of the functions $\mathcal{A}, \mathcal{B}, \mathcal{C}$ and $\mathcal{D}$, (3.12) must fulfill the following condition:

$$
\left|\begin{array}{cc}
-\frac{1}{\sqrt{f(r)}} \partial_{t} S_{\uparrow}(t, r)+m & -\sqrt{\frac{f(r)}{A(r)}} \partial_{r} S_{\uparrow}(t, r) \\
\sqrt{\frac{f(r)}{A(r)}} \partial_{r} S_{\uparrow}(t, r) & \frac{1}{\sqrt{f(r)}} \partial_{t} S_{\uparrow}(t, r)+m
\end{array}\right|=0 .
$$

Writing the action as an expansion in a power series of $\left(\frac{\hbar}{i}\right)$,

$$
S_{\uparrow}(t, r)=S_{0 \uparrow}(t, r)+\left(\frac{\hbar}{i}\right) S_{1 \uparrow}(t, r)+\left(\frac{\hbar}{i}\right)^{2} S_{2 \uparrow}(t, r)+\ldots
$$

and making use of the WKB approximation (we neglect terms of order $\left(\frac{\hbar}{i}\right)$ and higher); we finally obtain a nonlinear first-order partial differential equation which corresponds to the Hamilton-Jacobi equation of motion to the leading order in the action $S_{\uparrow}$,

$$
-A(r)\left(\frac{\partial S_{0 \uparrow}(t, r)}{\partial t}\right)^{2}+f(r)^{2}\left(\frac{\partial S_{0 \uparrow}(t, r)}{\partial r}\right)^{2}+A(r) f(r) m^{2}=0 .
$$

Previously, in [12], it was obtained the same Hamilton-Jacobi equation corresponding, in that case, to the propagation of a massless scalar field. The leading-order action solution is

$$
S_{0 \uparrow}(t, r)=-\omega t \pm \omega \int_{r_{\text {in }}}^{r_{\text {out }}} \frac{\sqrt{A(r)}}{f(r)} d r
$$

The plus/minus sign corresponds to ingoing/outgoing fermions, respectively; $r_{\text {in }}$ and $r_{\text {out }}$ corresponds to a position inside and outside of the black hole, respectively; and $\omega$ is the energy of the emitted or absorbed fermion. Then, performing the complex integration of (3.17) and using the saddle point approximation for the amplitude, $K\left(r_{\text {out }}, t_{2} ; r_{\text {in }}, t_{1}\right)=$ $N \exp \left[\frac{i}{\hbar} S_{0}\left(r_{\text {out }}, t_{2} ; r_{\text {in }}, t_{1}\right)\right]$, we obtain the tunneling emission probability,

$$
P_{e} \sim \exp \left[-\frac{\pi}{\hbar} \omega \sqrt{N}\right]
$$

where we can identify the Hagedorn temperature of LST as

$$
T_{H}=\frac{\hbar m_{s}}{2 \pi \sqrt{N}}
$$

$m_{s}$ being the string mass.

We have found that the same results for the massless scalar particles in [12] are obtained here for fermions. So, we can conclude that the tunneling emission through the event horizon of the LST does not depend on the particle's spin. 
We would like to consider briefly the case for massive particles. If we solve (3.16) by taking into account the mass term, we obtain the following action at leading order:

$$
S_{0 \uparrow}(t, r)=-\omega t \pm \int_{r_{\text {in }}}^{r_{\text {out }}} \frac{\sqrt{A(r)}}{f(r)} \sqrt{\omega^{2}-f(r) m^{2}} d r .
$$

We notice that one obtains again the same results for the emission probability and temperature, thus the emission does not depend on the mass of the emitted particle.

\subsection{FERMion MODES AND BACKSCATTERING SPECTRUM}

We are interested in calculating the average number of emitted fermions as well as the radiation flux. In [26], the authors calculated the Hawking blackbody spectrum corresponding to a spherically symmetric static black hole. It is our aim to perform a similar analysis corresponding to LST black hole. Our starting point is the two-dimensional action (3.17), which can be written as

$$
S_{0}(r, t)=\omega\left(t \pm r^{*}\right),
$$

where $r^{*}$ is the tortoise coordinate defined as

$$
d r^{*}=\frac{\sqrt{A(r)}}{f(r)} d r
$$

Then if we consider the outgoing/ingoing null coordinates

$$
u=t-r^{*}, \quad v=t+r^{*},
$$

we can define the right/left modes inside and outside of the black hole in the following way:

$$
\begin{aligned}
& \phi_{\text {in }}^{R}=e^{-\frac{i}{\hbar} \omega u_{\text {in }}}, \quad \phi_{\text {in }}^{L}=e^{-\frac{i}{\hbar} \omega v_{\text {in }}} \\
& \phi_{\text {out }}^{R}=e^{-\frac{i}{\hbar} \omega u_{\text {out }}}, \quad \phi_{\text {out }}^{L}=e^{-\frac{i}{\hbar} \omega v_{\text {out }}} .
\end{aligned}
$$

The Kruskal coordinates, see e.g. [27], corresponding to the inside and outside of the LST black hole are defined as

$$
\begin{aligned}
& T_{\text {in }}=e^{\kappa r_{\text {in }}^{*}} \cosh \left(\kappa t_{\text {in }}\right), \quad X_{\text {in }}=e^{\kappa r_{\text {in }}^{*}} \sinh \left(\kappa t_{\text {in }}\right), \\
& T_{\text {out }}=e^{\kappa r_{\text {out }}^{*}} \sinh \left(\kappa t_{\text {out }}\right), \quad X_{\text {out }}=e^{\kappa r_{\text {out }}^{*}} \cosh \left(\kappa t_{\text {out }}\right),
\end{aligned}
$$

where $\kappa$ is the surface gravity and is related with the Hawking temperature through the

relation $T_{H}=\frac{\hbar \kappa}{2 \pi}$. The two sets of Kruskal coordinates are then connected by the following transformation relation between the coordinates $t$ and $r$ :

$$
t_{\text {in }} \rightarrow t_{\text {out }}-i \frac{\pi}{2 \kappa}, \quad r_{\text {in }}^{*} \rightarrow r_{\text {out }}^{*}+i \frac{\pi}{2 \kappa}
$$


Moreover, the null coordinates are also transformed as

$$
u_{\text {in }} \rightarrow u_{\text {out }}-i \frac{\pi}{\kappa}, \quad v_{\text {in }} \rightarrow v_{\text {out }} .
$$

Eventually, we have obtained a transformation relation between the left/right modes inside and outside the black hole,

$$
\phi_{\text {in }}^{R} \rightarrow \phi_{\text {out }}^{R} e^{-\frac{\pi \omega}{\hbar \kappa}}, \quad \phi_{\text {in }}^{L} \rightarrow \phi_{\text {out }}^{L} .
$$

As a comment, we note that this last relation is precisely the relation that one obtains between the Bogoliubov coefficients in the standard study of the emission of Hawking radiation.

Now, we want to calculate the average number of fermions emitted by the black hole. We construct the physical state associated to a system of $n$ number of a noninteracting virtual pair of fermions created inside the black hole,

$$
|\psi\rangle=N \sum_{n}\left|n_{i n}^{L}\right\rangle \otimes\left|n_{i n}^{R}\right\rangle .
$$

Since outside of the black hole we can carry out observations, we want to write the physical state in terms of the out eigenstates. Thus using the relation (3.28) between the modes inside and outside of the black hole, we can obtain the desired expression,

$$
|\psi\rangle=N \sum_{n} e^{-\frac{\pi \omega n}{\hbar \kappa}}\left|n_{\text {out }}^{L}\right\rangle \otimes\left|n_{\text {out }}^{R}\right\rangle .
$$

In order to calculate the normalization constant $N$, we make use of the orthonormalization condition between two orthonormalized states,

$$
\left\langle\psi_{m} \mid \psi_{n}\right\rangle=\delta_{m n} .
$$

Then, considering two states $\left|\psi_{n}\right\rangle$ and $\left\langle\psi_{m}\right|$, we construct

$$
\begin{aligned}
\left\langle\psi_{m} \mid \psi_{n}\right\rangle & =\left(N \sum _ { m } e ^ { - \frac { \pi \omega m } { \hbar \kappa } } \left\langlem_{\text {out }}^{L}\left|\otimes\left\langle m_{\text {out }}^{R}\right|\right) \cdot\left(N \sum_{n} e^{-\frac{\pi \omega n}{\hbar \kappa}}\left|n_{\text {out }}^{L}\right\rangle \otimes\left|n_{\text {out }}^{R}\right\rangle\right)\right.\right. \\
& =N^{2} \sum_{m, n} e^{-\frac{\pi \omega(m+n)}{\hbar \kappa}}\left\langle m_{\text {out }}^{L} \mid n_{\text {out }}^{L}\right\rangle \otimes\left\langle m_{\text {out }}^{R} \mid n_{\text {out }}^{R}\right\rangle
\end{aligned}
$$

and taking into account (3.31), we obtain the following relation:

$$
1=N^{2} \sum_{n} e^{-\frac{2 \pi \omega n}{\hbar \kappa}},
$$

from which we obtain the normalization constant corresponding to fermions $(n=0,1)$,

$$
N_{f}=\left(1+e^{-\frac{2 \pi \omega}{\hbar \kappa}}\right)^{-\frac{1}{2}} .
$$


A state associated to a system of fermions inside the black hole can be written as

$$
\left|\psi_{f}\right\rangle=\left(1-e^{+\frac{2 \pi \omega}{\hbar \kappa}}\right)^{\frac{1}{2}} \sum_{n} e^{-\frac{\pi \omega n}{\hbar \kappa}}\left|n_{\text {out }}^{L}\right\rangle \otimes\left|n_{\text {out }}^{R}\right\rangle .
$$

We might construct the density matrix operator for a system of fermions as

$$
\begin{aligned}
\rho_{f} & =\left|\psi_{n}\right\rangle\left\langle\psi_{m}\right| \\
& =\left(N_{f} \sum_{n} e^{-\frac{\pi \omega n}{\hbar \kappa}}\left|n_{\text {out }}^{L}\right\rangle \otimes\left|n_{\text {out }}^{R}\right\rangle\right) \cdot\left(N_{f} \sum_{m} e^{-\frac{\pi \omega m}{\hbar \kappa}}\left\langle m_{\text {out }}^{L}\right| \otimes\left\langle m_{\text {out }}^{R}\right|\right) \\
& =\left(1-e^{-\frac{2 \pi \omega}{\hbar \kappa}}\right) \sum_{n, m} e^{-\frac{\pi \omega(n+m)}{\hbar \kappa}}\left(\left|n_{\text {out }}^{L}\right\rangle \otimes\left|n_{\text {out }}^{R}\right\rangle\right) \cdot\left(\left\langle m_{\text {out }}^{L}\right| \otimes\left\langle m_{\text {out }}^{R}\right|\right) \\
& =\left(1-e^{-\frac{2 \pi \omega}{\hbar \kappa}}\right) \sum_{n, m} e^{-\frac{\pi \omega(n+m)}{\hbar \kappa}}\left(\left|n_{\text {out }}^{L}\right\rangle\left\langle m_{\text {out }}^{L}\right|\right) \otimes\left(\left|n_{\text {out }}^{R}\right\rangle\left\langle m_{\text {out }}^{R}\right|\right) .
\end{aligned}
$$

Tracing over the left modes, $\left\langle m_{\text {out }}^{L}\left|\left(\left|n_{\text {out }}^{L}\right\rangle\left\langle m_{\text {out }}^{L}\right|\right)\right| n_{\text {out }}^{L}\right\rangle$, and taking into account the orthonormalization condition (3.31), we obtain

$$
\rho_{f}^{R}=\left(1-e^{+\frac{2 \pi \omega}{\hbar \kappa}}\right) \sum_{n} e^{-\frac{2 \pi \omega n}{\hbar \kappa}}\left|n_{\text {out }}^{R}\right\rangle\left\langle n_{\text {out }}^{R}\right| .
$$

This expression corresponds to the density matrix of fermions in terms of the right outgoing modes. These modes will be detected at asymptotic infinity as the Hawking radiation. Finally, using the equation

$$
\begin{aligned}
\left\langle n_{f}\right\rangle & =\operatorname{Tr}\left(n \cdot \rho_{f}^{R}\right) \\
& =\left(1-e^{+\frac{2 \pi \omega}{\hbar \kappa}}\right) \sum_{n} n \cdot e^{-\frac{2 \pi \omega n}{\hbar \kappa}}\left|n_{\text {out }}^{R}\right\rangle\left\langle n_{\text {out }}^{R}\right|,
\end{aligned}
$$

tracing over the right outgoing modes, $\left\langle m_{\text {out }}^{R}\left|\left(\left|n_{\text {out }}^{R}\right\rangle\left\langle n_{\text {out }}^{R}\right|\right)\right| n_{\text {out }}^{R}\right\rangle$, and taking into account the orthonormalization condition (3.31), we obtain the average number of fermions detected at asymptotic infinity,

$$
\left\langle n_{f}\right\rangle=\left(1-e^{+\frac{2 \pi \omega}{\hbar \kappa}}\right) \sum_{n} n \cdot e^{-\frac{2 \pi \omega n}{\hbar \kappa}}=\frac{1}{e^{\frac{2 \pi \omega}{\hbar \kappa}}+1} .
$$

Integrating this last expression over all energy ranges, we obtain the flux of fermions seen by an asymptotic observer,

$$
F_{\infty}=\frac{1}{2 \pi} \int_{0}^{\infty}\left\langle n_{f}\right\rangle \omega d \omega=\frac{\hbar^{2} \kappa^{2}}{96 \pi}=\frac{\pi}{24} T_{H}^{2},
$$

where $T_{H}$ is the temperature defined in (3.19). 
It is well known that some emitted radiation will be partially scattered back to the event horizon. This fact is due to the gravitational potential barrier around the black hole where some fraction of radiation is reflected back to the hole, acting thus as a filter for the emitted radiation. In this way, Eq. (3.39) must be modified to the following new expression:

$$
\left\langle n_{f}\right\rangle=\frac{\Gamma_{\omega l}}{e^{\frac{2 \pi \omega}{\hbar \kappa}}+1}
$$

where $\Gamma_{\omega l}$ is the greybody factor and it accounts for the deviation from pure Planckian spectrum. Henceforth, we will consider the value $\Gamma_{\omega 0}$ since the main contribution to the greybody factor comes from the zero angular momentum $l=0$ whenever the relation $\omega M \ll$ 1 is fulfilled.

Greybody factors have a relevant importance because a successful microscopic account of black hole thermodynamics should be able to predict them. For example, it is shown in [28] that D-branes provide an account of black hole microstates which is successful to predict the greybody factors. There exists a vast literature on how to compute greybody factors in the context of the quantum field theory in curved space-time, e.g. [29, 30, 31, 32, 33, 34, 35].

Using the solution corresponding to the propagation of a massless scalar field in the background of LST, see [36], and applying the usual matching techniques described in the previous references, we have verified that the greybody factor is 1 . One could expect this value since the pure-thermal behavior characterizes the LST emission. Likewise, we expect to obtain identical results for the emission of fermions in LST. Then, we are going to find the fermion modes of the Dirac equation (3.9) corresponding to the two-dimensional LST background (3.8), and next using the matching technique we will compute the greybody factor.

Now, in order to study the Dirac equation, we choose the following basis for the spinor field:

$$
\Psi(t, r)=\left(\begin{array}{c}
\Psi_{+}(t, r) \\
\Psi_{-}(t, r)
\end{array}\right)
$$

considering that each term is a two-component spin-up and spin-down spinor, $\Psi_{+}=\left(\begin{array}{c}\Psi_{+}^{\uparrow} \\ \Psi_{+}^{\downarrow}\end{array}\right)$ and $\Psi_{-}=\left(\begin{array}{c}\Psi_{-}^{\downarrow} \\ \Psi_{-}^{\uparrow}\end{array}\right)$. Using this basis for the spinor field and the $\gamma^{0}$ and $\gamma^{1}$ matrices defined in (3.4), we obtain two sets of equivalent equations corresponding to the spin-up and spindown fermion case. We will study the spin-up fermion case; equivalently we could also study 
the spin-down case. Therefore, the Dirac equation becomes

$$
\begin{aligned}
& \left(\frac{-i}{\sqrt{f(r)}} \partial_{t}+m\right) \Psi_{+}(t, r)-i\left(\sqrt{\frac{f(r)}{A(r)}} \partial_{r}+\frac{f^{\prime}(r)}{4 \sqrt{A(r) f(r)}}\right) \Psi_{-}(t, r)=0, \\
& \left(\frac{i}{\sqrt{f(r)}} \partial_{t}+m\right) \Psi_{-}(t, r)+i\left(\sqrt{\frac{f(r)}{A(r)}} \partial_{r}+\frac{f^{\prime}(r)}{4 \sqrt{A(r) f(r)}}\right) \Psi_{+}(t, r)=0 .
\end{aligned}
$$

Next, we consider the following ansatz for the spinor field:

$$
\Psi_{+}(t, r)=\phi_{+}(r) e^{-i \omega t}, \quad \Psi_{-}(t, r)=i \phi_{-}(r) e^{-i \omega t}
$$

Substituting this expressions into (3.43) and after doing algebra we obtain the following set of equations:

$$
\begin{aligned}
& \partial_{r} \phi_{-}(r)+\frac{f(r)^{\prime}}{4 f(r)} \phi_{-}(r)+\left(m \sqrt{\frac{A(r)}{f(r)}}-\omega \frac{\sqrt{A(r)}}{f(r)}\right) \phi_{+}(r)=0, \\
& \partial_{r} \phi_{+}(r)+\frac{f(r)^{\prime}}{4 f(r)} \phi_{+}(r)+\left(m \sqrt{\frac{A(r)}{f(r)}}+\omega \frac{\sqrt{A(r)}}{f(r)}\right) \phi_{-}(r)=0 .
\end{aligned}
$$

This set of coupled equations is analytically solvable. If we define

$$
\eta_{ \pm}(r) \equiv m \sqrt{\frac{A(r)}{f(r)}} \pm \omega \frac{\sqrt{A(r)}}{f(r)}
$$

we obtain the following second-order differential equation:

$$
\begin{aligned}
& \eta_{+}^{-1}(r) \phi_{+}(r)^{\prime \prime}+\left(\partial_{r} \eta_{+}^{-1}(r)+\eta_{+}^{-1}(r) \frac{f(r)^{\prime}}{2 f(r)}\right) \phi_{+}(r)^{\prime}+ \\
& +\left(\partial_{r} \eta_{+}^{-1}(r) \frac{f(r)^{\prime}}{4 f(r)}+\eta_{+}^{-1}(r) \partial_{r}\left(\frac{f(r)^{\prime}}{4 f(r)}\right)+\eta_{+}^{-1}(r)\left(\frac{f(r)^{\prime}}{4 f(r)}\right)^{2}-\eta_{-}(r)\right) \phi_{+}(r)=0 .
\end{aligned}
$$

In order to simplify the resolution of the above equations, we consider the propagation of a massless fermion. Substituting the values of $f(r)$ and $A(r)$ given in (2.3) into (3.46) and (3.47), eventually we obtain the propagation equation for a massless fermion mode,

$$
4 r^{2}\left(r^{2}-r_{0}^{2}\right)^{2} \phi_{+}(r)^{\prime \prime}+4 r\left(r^{2}-r_{0}^{2}\right)\left(r^{2}+2 r_{0}^{2}\right) \phi_{+}(r)^{\prime}+\left(4 \omega^{2} N r^{4}-4 r_{0}^{2} r^{2}+5 r_{0}^{4}\right) \phi_{+}(r)=0 .
$$

This equation admits the following solution:

$$
\phi_{+}(r)=\sqrt{r}\left(r^{2}-r_{0}^{2}\right)^{-1 / 4}\left(C_{1}\left(r^{2}-r_{0}^{2}\right)^{-\frac{i}{2} \omega \sqrt{N}}+\frac{C_{2}}{2 i \omega \sqrt{N}}\left(r^{2}-r_{0}^{2}\right)^{\frac{i}{2} \omega \sqrt{N}}\right),
$$


where $C_{1}$ and $C_{2}$ are arbitrary constants.

Next, following the matching recipe [30, 31, 32, 33], we must match both solutions at asymptotic infinity and at near the event horizon in a matching point defined as $r_{m}$. We must calculate the flux defined as

$$
\mathcal{F}=\frac{1}{2 i}\left(\phi_{+}^{*}(r) r^{3} f(r) \partial_{r} \phi_{+}(r)-c . c .\right) .
$$

The ratio of the flux evaluated at the near horizon and at the asymptotic infinity, respectively, is the absorption cross section, which can be demonstrated that, in the low-energy regime, it is equal to the greybody factor. The mode solution at the near horizon limit is obtained imposing the propagation of ingoing modes as a boundary condition. Then, if we expand the solution (3.49) near the horizon, we obtain

$$
\phi_{h}(r)=C_{h}\left(r-r_{0}\right)^{-\frac{1}{4}-\frac{i}{2} \omega \sqrt{N}}
$$

where we have collected all the terms that are independent of the $r$ coordinate in the constant $C_{h}$. The corresponding flux is

$$
\mathcal{F}_{h}=\frac{\left|C_{h}\right|^{2}}{2} \omega \sqrt{N} \frac{r\left(r+r_{0}\right)}{\sqrt{r-r_{0}}} .
$$

Next, we calculate the mode solution at the asymptotic limit. We must take into account that in this limit the metric function $f(r)$ fulfills the relation

$$
\lim _{r \rightarrow \infty} f(r)=1
$$

Solving Eq. (3.47) for the massless case using (3.53), we obtain the following mode solution at the asymptotic limit:

$$
\phi_{\infty}(r)=C_{\infty} r^{i \omega \sqrt{N}}
$$

Therefore, the corresponding flux is

$$
\mathcal{F}_{\infty}=\frac{\left|C_{\infty}\right|^{2}}{2} \omega \sqrt{N} r^{2} .
$$

In order to find a relation between the constants $C_{h}$ and $C_{\infty}$, we match both solutions at the matching point $r_{m}$, which fulfills $r_{0}<<r_{m}$. Hence, imposing the matching condition, we find the following relation between the constants:

$$
\left|C_{\infty}\right|^{2}=\frac{\left|C_{h}\right|^{2}}{\sqrt{r_{m}-r_{0}}} .
$$


Finally, if we calculate the greybody factor as the ratio of the ingoing flux through the horizon, $\mathcal{F}_{h}$, to the outgoing flux at the asymptotic limit, $\mathcal{F}_{\infty}$, we obtain

$$
\Gamma_{\omega} \equiv \frac{\left|\mathcal{F}_{h}\right|}{\left|\mathcal{F}_{\infty}\right|}=1
$$

This result indicates that for LST we obtain a pure Planckian spectrum. Effectively, one would expect this result since we have demonstrated in [11, 12] how LST exhibits a purely thermal behavior, even taking into account the backreaction of the metric.

\section{Conclusion}

In previous works the emission of massless scalar particles in the LST background had been studied; however, it is interesting to extend this study to the emission of Dirac particles in order to complete the emission description in LST.

We have calculated the emission of a fermion field in a two-dimensional effective LST metric relevant for the emission process. Interestingly, we also have found that the emission shows a purely thermal profile as in the scalar case. This lead us to the conclusion that the thermal profile of LST is independent of the emitted fields; the independent mass temperature (3.19) is the clue for this behavior.

The gravitational potential barrier around the black hole acts as a filter for the emitted radiation, therefore the spectrum detected at the asymptotic infinity is not a pure Planckian spectrum. The greybody factor accounts for this deviation from the purely blackbody spectrum. However we have verified that LST exhibits a different behavior; the independence of its temperature on the black hole mass leads to the fact that the emission is purely thermal, even taking into account backreaction effects. Therefore, one expects that the spectrum shall be purely Planckian and the greybody factor takes the value 1 . We have verified this last assumption both for the emission of scalar particles and the emission of fermions in a two-dimensional effective metric.

Even if one considers backreaction and self-gravitating effects, the flux (3.40) remains fixed at the Hagedorn temperature (3.19). Thus, the results obtained in this work, corresponding to the emission of fermions in LST background, match the same conclusions concerning the information loss paradox in scalar field emission studied in Refs. [11, 12]. Hence, this work completes the study concerning the emission of different kind of fields in LST. 


\section{Acknowledgments:}

I thank Pere Talavera for discussions on this work.

\section{REFERENCES}

[1] S. W. Hawking, "Particle Creation by Black Holes," Commun. Math. Phys. 43 (1975) 199-220.

[2] J. M. Maldacena, "Black holes in string theory," hep-th/9607235.

[3] A. W. Peet, "TASI lectures on black holes in string theory," hep-th/0008241.

[4] S. R. Das and S. D. Mathur, "The quantum physics of black holes: Results from string theory," Ann. Rev. Nucl. Part. Sci. 50 (2000) 153 [gr-qc/0105063].

[5] L. Susskind and J. Lindesay, "An introduction to black holes, information and the string theory revolution: The holographic universe," Hackensack, USA: World Scientific (2005) $183 \mathrm{p}$

[6] M. K. Parikh, F. Wilczek, "Hawking radiation as tunneling," Phys. Rev. Lett. 85 (2000) 5042-5045. hep-th/9907001.

[7] P. Kraus and F. Wilczek, "Selfinteraction correction to black hole radiance," Nucl. Phys. B 433 (1995) 403 gr-qc/9408003.

[8] M. K. Parikh, "Energy conservation and Hawking radiation," hep-th/0402166.

[9] M. Arzano, A. J. M. Medved and E. C. Vagenas, "Hawking radiation as tunneling through the quantum horizon," JHEP 0509 (2005) 037 hep-th/0505266].

[10] B. Zhang, Q. -y. Cai, L. You and M. -s. Zhan, "Hidden Messenger Revealed in Hawking Radiation: A Resolution to the Paradox of Black Hole Information Loss," Phys. Lett. B 675 (2009) 98 [arXiv:0903.0893 [hep-th]].

[11] O. Lorente-Espin, P. Talavera, "A Silence black hole: Hawking radiation at the Hagedorn temperature," JHEP 0804 (2008) 080. arXiv:0710.3833 [hep-th]].

[12] O. Lorente-Espin, "Some considerations about NS5 and LST Hawking radiation," Phys. Lett. B 703 (2011) 627 [arXiv:1107.0713 [hep-th]]. 
[13] D. Kutasov, "Introduction to little string theory," Lectures given at the Spring School on Superstrings and Related Matters. Trieste, 2-10 April 2001.

[14] O. Aharony, "A Brief review of 'little string theories'," Class. Quant. Grav. 17 (2000) 929-938. [hep-th/9911147].

[15] A. Kapustin, "On the universality class of little string theories," Phys. Rev. D63 (2001) 086005. hep-th/9912044.

[16] D. Kutasov, D. A. Sahakyan, "Comments on the thermodynamics of little string theory," JHEP 0102 (2001) 021. hep-th/0012258.

[17] M. Rangamani, "Little string thermodynamics," JHEP $0106 \quad$ (2001) 042. hep-th/0104125.

[18] O. Aharony, M. Berkooz, D. Kutasov and N. Seiberg, "Linear dilatons, NS five-branes and holography," JHEP 9810 (1998) 004 hep-th/9808149.

[19] A. W. Peet, J. Polchinski, "UV / IR relations in AdS dynamics," Phys. Rev. D59 (1999) 065011. hep-th/9809022.

[20] N. Seiberg, "New theories in six-dimensions and matrix description of M theory on $\mathrm{T}^{* *} 5$ and T**5 / Z(2)," Phys. Lett. B408 (1997) 98-104. hep-th/9705221].

[21] J. M. Maldacena, A. Strominger, "Semiclassical decay of near extremal five-branes," JHEP 9712 (1997) 008. hep-th/9710014.

[22] D. Kutasov, N. Seiberg, "Noncritical superstrings," Phys. Lett. B251 (1990) 67-72.

[23] D. R. Brill and J. A. Wheeler, Rev. Mod. Phys. 29 (1957) 465.

[24] H. T. Cho, Phys. Rev. D 68 (2003) 024003 [gr-qc/0303078.

[25] R. Kerner and R. B. Mann, Class. Quant. Grav. 25 (2008) 095014 arXiv:0710.0612 [hep-th]].

[26] R. Banerjee, B. R. Majhi, "Hawking black body spectrum from tunneling mechanism," Phys. Lett. B675 (2009) 243-245. arXiv:0903.0250 [hep-th]].

[27] C. W. Misner, K. S. Thorne and J. A. Wheeler, "Gravitation," San Francisco 1973, $1279 \mathrm{p}$ 
[28] S. R. Das and S. D. Mathur, "Comparing decay rates for black holes and D-branes," Nucl. Phys. B 478 (1996) 561 [hep-th/9606185].

[29] J. A. H. Futterman, F. A. Handler and R. A. Matzner, "Scattering From Black Holes," Cambridge, UK: Univ. Pr. (1988) 192 P. (Cambridge Monographs on Mathematical Physics)

[30] J. M. Maldacena and A. Strominger, "Black hole grey body factors and d-brane spectroscopy," Phys. Rev. D 55 (1997) 861 [hep-th/9609026].

[31] S. S. Gubser and I. R. Klebanov, "Four-dimensional greybody factors and the effective string," Phys. Rev. Lett. 77 (1996) 4491 hep-th/9609076].

[32] I. R. Klebanov and M. Krasnitz, "Fixed scalar greybody factors in five-dimensions and four-dimensions," Phys. Rev. D 55 (1997) 3250 [hep-th/9612051].

[33] I. R. Klebanov and S. D. Mathur, "Black hole greybody factors and absorption of scalars by effective strings," Nucl. Phys. B 500 (1997) 115 [hep-th/9701187].

[34] S. S. Gubser, I. R. Klebanov and A. A. Tseytlin, "String theory and classical absorption by three-branes," Nucl. Phys. B 499 (1997) 217 [hep-th/9703040].

[35] A. W. Peet, "The Bekenstein formula and string theory (N-brane theory)," Class. Quant. Grav. 15 (1998) 3291 hep-th/9712253].

[36] K. Narayan and M. Rangamani, "Hot little string correlators: A View from supergravity," JHEP 0108 (2001) 054 hep-th/0107111]. 